\title{
Quantum Yang-Mills on the Two-Sphere
}

\author{
Dana S. Fine \\ Department of Mathematics, Southeastern Massachusetts University, North Dartmouth, \\ MA 02747, USA
}

Received December 21, 1989; in revised form March 16, 1990

\begin{abstract}
We obtain the quantum expectations of gauge-invariant functions of the connection on a principal $G=S U(N)$ bundle over $S^{2}$. We show that the space $\mathscr{A} / \mathscr{G}_{m}$ of connections modulo gauge transformations which are the identity at one point is itself a principal bundle over $\Omega G$, based loops in the symmetry group. The fiber in $\mathscr{A} / \mathscr{G}_{m}$ is an affine linear space. Quantum expectations are iterated path integrals first over this fiber then over $\Omega G$, each with respect to the push-forward to $\mathscr{A} / \mathscr{G}_{m}$ of the measure $e^{-S(A)} \mathscr{D} A$. $S(A)$ denotes the Yang-Mills action on $\mathscr{A}$. There is a global section of $\mathscr{A} / \mathscr{G}_{m}$ on which the first integral is a Gaussian. The resulting measure on $\Omega G$ is the conditional Wiener measure. We explicitly compute the expectations of a special class of Wilson loops.
\end{abstract}

\section{Introduction}

We consider the expectation of a gauge-invariant function $f$ with respect to the formal measure $\int_{\mathscr{A}} e^{-S(A)} \mathscr{D} A$, where $S(A)=\frac{1}{4}\left\|F_{A}\right\|^{2}$ and $\mathscr{A}$ is the space of all connections on a $G=S U(N)$ (trivial) bundle over $S^{2}$. This measure pushes forward under the projection $\mathscr{A} \rightarrow \mathscr{A} / \mathscr{G}_{m}$, where $\mathscr{G}_{m}$ is the space of gauge transformations which are the identity at a given point $m \in S^{2}$. The push-forward measure formally defines a measure $\mu$ which differs from the natural measure on $\mathscr{A} / \mathscr{G}_{m}$ by a factor describing how the size of the orbit varies within $\mathscr{A} / \mathscr{G}_{m}$. The devices of gauge-fixing and Faddeev-Popov ghosts give a presumably well-defined measure on $\mathscr{A}$, whose push-forward agrees with $\mu$. This agreement permits us to compute the expectation of $f$ directly on $\mathscr{A} / \mathscr{G}_{m}$ with respect to the measure $\mu$.

The space $\mathscr{A} / \mathscr{G}_{m}$ is homotopic to $\Omega G$, based loops on $G$ as shown in Atiyah and Jones [1] and Singer [2]. Section 2.1 presents the homotopy equivalence via a map $\xi: \mathscr{A} / \mathscr{G}_{m} \rightarrow \Omega G$. In fact, $\xi$ is the projection map of the bundle $\mathscr{A} / \mathscr{G}_{m}$ over $\Omega G$ with an affine space as the fiber.

Integration on $\mathscr{A} / \mathscr{G}_{m}$ is integration over the affine fibers followed by integration over $\Omega G$, with respect to the measures $\mu$ induces. We exhibit the measure on each fiber as a Gaussian. Integrating over the fibers defines the push-forward measure 
$\xi_{*} \mu$. We show $\xi_{*} \mu$ is the Wiener measure on $\Omega G$, written as $e^{-(1 / 8)\|\dot{\gamma}\|^{2}} \mathscr{D} \gamma$. Thus, we interpret the integral $\int_{\mathscr{A}} f(A) e^{-S(A)} \mathscr{D} A$ to mean: integrate $f$ over each fiber, using the Gaussian measure, to define a function $\hat{f}$ on $\Omega G$, then evaluate $\int_{\Omega G} \hat{f}(\gamma) e^{-(1 / 8)\|\dot{\gamma}\|^{2}} \mathscr{D} \gamma$. We illustrate this procedure in the case $G=S U(2)$ with some special choices for the function $f$. In particular, if $\mathscr{W}$ denotes the Wilson loop given by parallel transport along a longitude from the north pole to the south pole of $S^{2}$ and back along a longitude at an equatorial angle $\phi$ relative to the first, and if $\chi_{\lambda}$ denotes the trace of $\mathscr{W}$ in the representation $\lambda$, we show the expectation is given by

$$
\left\langle\chi_{\lambda}(\mathscr{W})\right\rangle=\frac{\sum_{\mu} \sum_{\nu=|\lambda-\mu|}^{\lambda+\mu} \chi_{\mu}(1) \chi_{\nu}(1) e^{-\mu(\mu+1) \phi} e^{-v(v+1)(2 \pi-\phi)}}{\sum_{\mu} \chi_{\mu}(\mathbf{1}) \chi_{\mu}(1) e^{-\mu(\mu+1) 2 \pi}} .
$$

This extends readily to expectations of functions of products of Wilson loops.

After a brief section on notation, this paper proceeds as follows:

Section 1 presents the measure $\mu$ on $\mathscr{A} / \mathscr{G}_{m}$ and relates it to the gauge-fixed measure on $\mathscr{A}$ of the Faddeev-Popov approach.

Section 2 describes the map $\xi: \mathscr{A} / \mathscr{G}_{m} \rightarrow \Omega G$.

Section 3 exhibits the Gaussian measures on the fibers of $\mathscr{A} / \mathscr{G}_{m}$.

Section 4 links $\xi_{*} \mu$ to the Wiener measure.

Section 5 computes some examples.

\section{Conventions}

On a product bundle, a connection $A$ is a Lie-algebra-valued 1-form, and a gauge transformation $\psi$ is a $G$-valued function. The effect of a gauge transformation on a connection is

$$
\psi \cdot A=\psi^{-1} A \psi+\psi^{-1} \mathbf{d} \psi
$$

The horizontal lift according to a connection $A$ of a curve $\gamma(t)$ in $S^{2}$ is a $G$-valued function $U_{A}$ which satisfies the parallel transport equation along $\gamma$

$$
\frac{d}{d t} U_{A}(\gamma)=U_{A}(\gamma) A\left(\frac{d \gamma}{d t}\right)
$$

with $U_{A}(\gamma(0))=1$, the identity element in $G$. Parallel transport about a closed path in $S^{2}$ is a Wilson loop. If the closed path begins at the north pole, where the gauge transformation are the identity, the corresponding Wilson loops are gauge invariant.

The curvature of a connection is a Lie-algebra-valued 2-form $F_{A}$. The $G$-invariant metric on the Lie algebra combined with a metric on the base space defines a $G$-invariant metric (,) on Lie-algebra-valued forms. The Yang-Mills action $S(A)$ is the integral over the base space of the square in this metric of the curvature.

The metric on $\mathscr{A}$ induces a metric on $\mathscr{A} / \mathscr{G}_{m}$ by distinguishing a natural choice of representative in $T \mathscr{A}$ for each element of $T\left(\mathscr{A} / \mathscr{G}_{m}\right)$. Denoting the tangent to 
the gauge orbits by $T^{V} \mathscr{A}$, the metric on $A$ defines an orthogonal complement $T^{H} \mathscr{A}$. This distinguishes representatives in $T \mathscr{A}$ for elements of $T\left(\mathscr{A} / \mathscr{G}_{m}\right)$. The induced metric is the metric on $\mathscr{A}$ applied to these representatives. Explicitly, $T^{V} \mathscr{A}$ is the image of the covariant differential $D_{A}$ on Lie-algebra-valued functions which are 0 at the north pole. The orthogonal complement is $\operatorname{ker} D_{A}^{*}$, so the induced metric on $\mathscr{A} / \mathscr{G}_{m}$ is the metric on $\mathscr{A}$ restricted to $\operatorname{ker} D_{A}^{*}$.

\section{Path Integration and Gauge Invariance}

In general, the path integral computation for the expectation of a gauge-invariant function $f$, which, with the normalization constant $Z_{0}=\int_{\mathscr{A}} e^{-S(A)} \mathscr{D} A$, is the formal
integral

$$
\langle f\rangle=\frac{1}{Z_{0}} \int_{\mathscr{A}} e^{-S(A)} f(A) \mathscr{D} A,
$$

requires interpretation. Naively, the invariance of $S(A)$ under gauge transformations makes the integral over even the subset $\mathscr{G}_{m} \cdot A \subset \mathscr{A}(A$ some fixed element of $\mathscr{A})$ proportional to the volume of $\mathscr{G}_{m}$, which is infinite. We interpret the path integral (1.1) to mean the integral of the gauge-invariant function $f(A)$ over the space $\mathscr{A} / \mathscr{G}_{m}$ of inequivalent connections with the push-forward measure $\pi_{*}\left(e^{-S(A)} \mathscr{D} A\right)$, where

$$
\int_{O} \pi_{*}\left(e^{S(A)} \mathscr{D} A\right) \equiv \int_{\pi^{-1}(O)} e^{-S(A)} \mathscr{D} A
$$

for any open set $O \subset \mathscr{A} / \mathscr{G}_{m}$.

The push-forward measure $\pi_{*}\left(e^{-S(A)} \mathscr{D} A\right)$ is some Jacobian factor times $e^{-S(A)} \mathscr{D}_{\mathscr{G}_{m}} A$, where $\mathscr{D}_{\mathscr{G}_{m}} A$ is the natural measure on $\mathscr{A} / \mathscr{G}$. The natural measure corresponds to the metric on $\mathscr{A} / \mathscr{G}_{m}$ which the metric on $\mathscr{A}$ induces as above. See Moore and Nelson [3] or D'Hoker and Phong [4] for a discussion of the sense in which a metric determines a measure on an infinite-dimensional space.

In the finite-dimensional analogue of the above situation, the Jacobian depends only on the differential of the group action. For the analogue, replace $\mathscr{G}_{m}$ acting on $\mathscr{A}$ by a compact Lie group $H$ acting isometrically on a manifold $M$. In this case, the metric on $M$ defines a measure through the volume form $\mathrm{vol}_{M}$, as well as a metric on $M / H$ and its corresponding volume from $\operatorname{vol}_{M / H}$. The projection $\pi: M \rightarrow M / H$ pushes the measure on $M$ forward to the measure $\pi_{*}\left(\operatorname{vol}_{M}\right)$ on $M / H$, where

$$
\int_{M / H} f \pi_{*}\left(\operatorname{vol}_{M}\right)=\int_{M} f \circ \pi \operatorname{vol}_{M}
$$

That is, integration using $\pi_{*}\left(\operatorname{vol}_{M}\right)$ in $M / H$ is the same as integration over the orbits in $M$ using $\operatorname{vol}_{M}$. The differential of the action of $H$ at each point of $M$ defines a map $\alpha$ from the Lie algebra of $H$ to the tangent space of $M$ at that point. The Jacobian relating the push-forward measure to the natural measure $\operatorname{vol}_{M / H}$ proves to be the determinant of this map,

$$
\pi_{*}\left(\operatorname{vol}_{M}\right)=\operatorname{det}^{1 / 2}\left(\alpha^{*} \alpha\right) \operatorname{vol}_{M / H} .
$$

The right-hand side of (1.1) indicates an integration over the orbits in $\mathscr{A}$, so, by analogy, the Jacobian relating this to integration using $\mathscr{D}_{\mathscr{G}_{m}} A$ should be the 
determinant of the infinitesimal action of $\mathscr{G}_{m}$. The infinitesimal action, which maps Lie-algebra-vaued 0 -forms vanishing at the north pole to elements of $T^{V} \mathscr{A}$ is, again, the covariant derivative. Thus, up to a constant, the measure

$$
\mu=\operatorname{det}^{1 / 2}\left(D_{A}^{*} D_{A}\right) e^{-S(A)} \mathscr{D}_{\mathscr{G}_{m}} A,
$$

is $\pi_{*}(e-S(A) \mathscr{D} A)$. The constant reflects the fact that the volume of $\mathscr{G}_{m}$ is not unity. In Sect. 3, a slight modification of this analogy will yield the integration over those gauge orbits which constitute the affine fiber in $\mathscr{A} / \mathscr{G}_{m}$.

The above analogy is very loose. For instance, it treats $\mathscr{G}_{m}$ as though it were compact, with unit volume. In some sense we are factoring out the infinite volume of $\mathscr{G}_{m}$ from $\pi_{*}\left(e^{-S(A)} \mathscr{D} A\right)$ to leave the measure $\mu$. This analogy does agree with the Faddeev-Popov approach. Indeed, lifting $\mu$ to a measure on $\mathscr{A}$ via a gaugecondition and representing the overall determinant function as a fermionic integration yields the usual gauge-fixed measure on $\mathscr{A}$, as follows: Let $\mathscr{F}: \wedge^{1}(M, \mathbf{g}) \rightarrow$ $\wedge^{0}(M, \mathbf{g})$ be an admissible gauge. That is, for a fixed $A_{0} \in \mathscr{A}, A_{0}+\mathscr{F}^{-1}(0)$ is a slice, and $\left.\mathbf{d} \mathscr{F}\right|_{V}$, the restriction of the differential of $\mathscr{F}$ to the vertical directions, is $1-1$. To lift $\mu$ to a measure on $\mathscr{A}$, introduce a Gaussian integration over $\wedge^{0}(M, \mathbf{g})$, normalized to one,

$$
\begin{aligned}
\int_{\mathcal{O}} \mu & =\int_{\mathcal{O}} \mu \frac{1}{N_{\zeta}} \int_{\wedge^{0}} e^{-\xi\langle y, y\rangle} \mathscr{D} y \\
& =\frac{1}{N_{\zeta}} \int_{\mathcal{O}} \int_{\wedge^{0}} \operatorname{det}^{1 / 2}\left(D_{A}^{*} D_{A}\right) e^{-S(A)} e^{-\zeta\langle y, y\rangle} \mathscr{D}_{\mathscr{G}_{m}} A \mathscr{D} y,
\end{aligned}
$$

where $N_{\zeta}$ is a normalization constant depending on the parameter $\zeta$ and $\mathcal{O} \subset \mathscr{A} / \mathscr{G}_{m}$ is an arbitrary open set. The integration over $\wedge^{0}(M, \mathbf{g})$ is equivalent to an integration over $\mathscr{G}_{m}$ (at least perturbatively, or for sufficiently large $\zeta$ ). The resulting iterated integral over $\mathscr{G}_{m}$ and $\mathcal{O}$ corresponds under a change of variables given by $\mathscr{F}$ to an integral over $\pi^{-1}(\mathcal{O})$ as follows: The tangent to $\mathscr{A}$ decomposes as $T \mathscr{A}=T^{V} \mathscr{A} \oplus T^{H} \mathscr{A}$, with $T^{H} \mathscr{A} \sim T\left(\mathscr{A} / \mathscr{G}_{m}\right)$. Thus, $\left.\mathbf{d} \mathscr{F}\right|_{V}$ provides an isomorphism $T \mathscr{A} \sim \wedge^{0}(M, \mathbf{g}) \oplus T\left(\mathscr{A} / \mathscr{G}_{m}\right)$. The induced metric on $T\left(\mathscr{A} / \mathscr{G}_{m}\right)$ makes the map from $T^{H} \mathscr{A}$ to $T\left(\mathscr{A} / \mathscr{G}_{m}\right)$ an isometry, so the Jacobian for the change of variables is $\operatorname{det}^{1 / 2}\left(\left.\left.\mathbf{d} \mathscr{F}\right|_{V} ^{*} \mathbf{d} \mathscr{F}\right|_{V}\right)$. Thus, changing variables gives

$$
\int_{\mathscr{O}} \mu=\frac{1}{N_{\zeta}} \int_{\pi^{-1}(\mathcal{O})} \operatorname{det}^{1 / 2}\left(D_{A}^{*} D_{A}\right) e^{-S(A)} \operatorname{det}^{1 / 2}\left(\left.\left.\mathbf{d} \mathscr{F}\right|_{V} ^{*} \mathbf{d} \mathscr{F}\right|_{V}\right) e^{-\zeta\langle\mathscr{F}, \mathscr{F}\rangle} \mathscr{D} A .
$$

Assuming the determinants multiply as they would in finite dimensions, the product of determinants corresponds to det $\mathscr{M}$, where $\mathscr{M}=\left.\mathbf{d} \mathscr{F}\right|_{V}{ }^{\circ} D_{A}: \wedge^{0}(M, \mathbf{g}) \rightarrow \wedge^{0}(M, \mathbf{g})$. Finally, replacing det $\mathscr{M}$ with a fermionic integration, det $\mathscr{M}=\int e^{-\langle\bar{\psi}, \mathscr{M} \psi\rangle} \mathscr{D} \bar{\psi} \psi$, gives

$$
\int_{\mathscr{O}} \mu=\frac{1}{N_{\zeta}} \int_{\pi^{-1}(\mathcal{O})} \int e^{-[S(A)+\zeta\langle\mathscr{F}, \mathscr{F}\rangle+\langle\bar{\psi}, \mathscr{M} \psi\rangle]} \mathscr{D} \bar{\psi} \mathscr{D} \psi \mathscr{D} A .
$$

The right-hand side is the push-forward to $\mathscr{A} / \mathscr{G}_{m}$ of the standard gauge-fixed measure on $\mathscr{A}$.

The measure $\mu$ thus agrees with the gauge-fixing prescription as a measure on gauge-invariant functions. The above argument is closely-related to one Gawedzki [5] presents for Yang-Mills on $R^{4}$ in the case of space-like gauges. Having 
established the agreement between $\mu$ and the gauge-fixing prescription, we will work directly with the measure $\mu$, taking advantage of the homotopy equivalence between $\mathscr{A} / \mathscr{G}_{m}$ and $\Omega G$.

\section{From $\mathscr{A} / \mathscr{G}_{m}$ to $\Omega G$}

The construction of the map $\xi: \mathscr{A} / \mathscr{G}_{m} \rightarrow \Omega G$ is as follows. Introduce the usual spherical coordinates $(\theta, \phi)$ on $S^{2}$, choosing as the north pole $(\theta=0)$ the point at which all the gauge transformations are the identity. Now consider a family of closed curves each of which originates at the north pole, follows the longitude at equatorial angle 0 to the south pole, and returns to the north pole along the longitude at some equatorial angle $\phi$. Figure 2.1 illustrates one such curve. Let $\gamma_{A}(\phi)$ denote the parallel transport according to $A$ about the curve returning at angle $\phi$. The angle $\phi$ ranges from 0 to $2 \pi$, and $\gamma_{A}(0)=\gamma_{A}(2 \pi)$, so $\gamma_{A}(\phi)$ is the value at $\phi$ of a loop, $\gamma_{A} \in \Omega G$. Since $\gamma_{A}$ is parallel transport about a loop from the north pole, where the gauge transformations are the identity, $\gamma_{A}$ is gauge-invariant. Define the map $\xi$ by $\xi(A)=\gamma_{A}$. The gauge-invariance of $\gamma_{A}$ means $\xi$ depends only on the gauge-class of $A$, hence $\xi$ maps $\mathscr{A} / \mathscr{G}_{m}$ to $\Omega G$.

The image of $\xi$ will depend on which connections are in its range. The physically natural class of connections is the space of connections whose Yang-Mills action is finite. Notice these are also the connections on which $e^{-S(A)}$ is non-zero. As shown in Uhlenbeck [6], the space of finite-action connections is the Sobolev space of connections whose first derivatives are square-integrable. The corresponding class of gauge transformations are those whose second derivatives are square-integrable. In light of the Sobolev embedding theorem, $L_{k}^{p} \subset C^{l}$ for $p-\frac{\operatorname{dim} M}{k}>l$, not all connections on $S^{2}$ with finite action are continuous. The gauge transformations between them are continuous, but need not be $C^{1}$. Henceforth, $\mathscr{A}$ will refer to connections with finite Yang-Mills action, and $\mathscr{G}_{\text {and }} \mathscr{G}_{m}$ will refer to the gauge transformations which preserve this condition. Similarly, $\Omega G$ will refer to loops with finite energy.

Defining the usual (local) projection of a 1-form to its components by $\omega=P_{\theta} \omega \mathbf{d} \theta+P_{\phi} \omega \mathbf{d} \phi$ on $\omega \in \wedge^{1}(M, \mathbf{g})$,

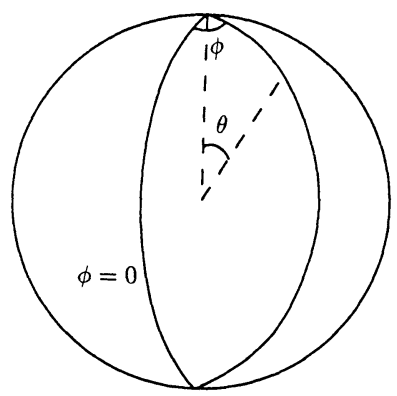

Fig. 2.1 
Theorem 2.1. $\mathscr{A} / \mathscr{G}_{m}$ is a principal bundle over $\Omega G$ with projection $\xi$ and fiber $\operatorname{ker} P_{\theta}$.

To prove this, we begin by showing that the image of $\xi$ is $\Omega G$. For any loop $\gamma \in \Omega G$, let $\ln \gamma \in \Omega \mathbf{g}$ be a choice for its logarithm. As a parametrized loop in $\mathbf{g}, \ln \gamma$ need not be continuous, even if $G$ is simply-connected. For example, in $S U(2)$, viewed as the sphere $S^{3}$, take $\gamma$ to be a great circle through the identity. Then, $\ln \gamma$ is a line through 0 in $s u(2)$, viewed as the tangent to the sphere. From 0 this line extends in both directions to $\infty$.

Nevertheless, consider any connection whose $\theta$-component is

$$
A_{\theta}=a(\theta) \ln \gamma,
$$

for some fixed function $a$ which is zero in sufficiently small neighborhoods of the north and south poles and satisfies $\int_{0}^{\pi} a=-1$. Such connections have parallel
transport along longitudes,

$$
U_{A}^{\phi}(\theta)=\exp \left\{\int_{0}^{\theta} a(\bar{\theta}) d \bar{\theta} \ln \gamma\right\} .
$$

Here $U_{A}^{\phi}(\theta)$ denotes parallel transport from the north pole an angle $\theta$ along the longitude at equatorial angle $\phi$. The corresponding loop is

$$
\xi(A)=U_{A}^{0}(\pi) U_{A}^{\phi}(\pi)^{-1}=\gamma .
$$

If $\ln \gamma$ is not continuous, neither is $A$. However, as will become clear in Sects. 3 and 4, there is a choice for the $\phi$-component of $A$ such that $S(A)=\frac{1}{8} \int_{S^{1}}\left(\gamma_{A}^{-1} \dot{\gamma}_{A}, \gamma_{A}^{-1} \dot{\gamma}_{A}\right)$, the energy action of the loop $\gamma_{A}$, which, by assumption, is finite. (The proof of this statement is strongly motivated by integration techniques which belong in the latter sections.) With this choice of $\phi$-component, $A$ is a connection with finite action such that $\xi(A)=\gamma$. This completes the proof that the image of $\xi$ is $\Omega G$.

To see that $\xi^{-1}(\gamma)$ is isomorphic to $\operatorname{ker} P_{\theta}$, let $A$ and $B$ be any two connections in the fiber over $\gamma$, so $\xi(A)=\xi(B)$. Consider the $G$-valued function

$$
\psi(\theta, \phi)=U_{A}^{\phi}(\theta)^{-1} U_{B}^{\phi}(\theta) .
$$

Notice these parallel transports involve only the $\theta$-components of the connections. Regarding $\psi$, which is the identity at the north pole, as a gauge transformation, the connections $B$ and $\psi \cdot A$ agree on parallel transport in the $\theta$-direction.

$$
U_{\psi \cdot A}^{\phi}=U_{A}^{\phi} \psi=U_{B}^{\phi} \text {. }
$$

The $\theta$-component of the difference of these connections must vanish. That is, there is a $\tau \in \operatorname{ker} P_{\theta}$ for which

$$
B=\psi \cdot(A+\tau) .
$$

Since $B$ was an arbitrary connection for which $\xi(B)=\xi(A)$,

$$
\xi^{-1}(\gamma) \subset\left\{[A+\tau]: \tau \in \operatorname{ker} P_{\theta}\right\} .
$$

Conversely, since the addition of $\tau \in \operatorname{ker} P_{0}$ has no effect on parallel transport in the $\theta$-direction, the inclusion also goes the other way, and

$$
\xi^{-1}(\gamma)=\left\{[A+\tau]: \tau \in \operatorname{ker} P_{\theta}\right\}
$$


Thus, for every $\gamma$ in any open set $\mathcal{O} \subset \Omega G$, a choice of $[A] \in \xi^{-1}(\gamma)$ determines an isomorphism between $\xi^{-1}(\gamma)$ and $\operatorname{ker} P_{\theta}$. Taken together these give an isomorphism between $\xi^{-1}(\mathcal{O})$ and $\mathcal{O} \times \operatorname{ker} P_{\theta}$, which completes the proof that $\mathscr{A} / \mathscr{G}_{m}$ is a principal bundle over $\Omega G$ with fiber ker $P_{\theta}$.

Under $\rho \circ \xi$, where $\rho$ denotes the projection $\rho: \mathscr{A} \rightarrow \mathscr{A} / \mathscr{G}_{m}$, the energy action on $\Omega G$ pulls back to a gauge-invariant function on $\mathscr{A}$. The energy action for a loop $\gamma_{A}=\rho \circ \xi(A)$ is the square norm of $\gamma_{A}^{-1} \dot{\gamma}_{A} \in \Omega T_{e} G \sim \Omega \mathrm{g}$. To relate this energy to the Yang-Mills action for the corresponding $A$, we use a covariant version of Stoke's law for a region of finite length and infinitesimal width. The product $\gamma_{A}(\phi)^{-1} \gamma_{A}(\phi+d \phi)$, which is $\mathbf{1}+\gamma_{A}^{-1} \dot{\gamma}_{A} d \phi$ to first order in $d \phi$, is also parallel transport about a closed path of infinitesimal width bounded by the longitudes at $\phi$ and $\phi+d \phi$. If the connection satisfies a limited continuity constraint, Stoke's law relates this parallel transport to the line integral of the curvature along the longitude at $\phi$.

Stoke's Law 2.2. For a connection $A$ whose $\phi$ - and $\theta$-components are continuous as a function of $\theta$ along each longitude and whose $\phi$-component vanishes at the north and south poles,

$$
\gamma_{A}(\phi)^{-1} \gamma_{A}(\phi+d \phi)=1-\int_{0}^{\pi} U_{A}^{\phi}(\theta) F_{\theta \phi}^{A}(\theta, \phi) U_{A}^{\phi}(\theta)^{-1} d \theta d \dot{\phi}
$$

to first order in $d \phi$.

The relation between the energy action as a function of $\gamma_{A} \in \Omega G$ and the curvature of the connection $A$ follows from

\section{Corollary 2.1.}

$$
\gamma_{A}^{-1} \dot{\gamma}_{A}=-\int_{0}^{\pi} U_{A}^{\phi} F_{\theta \phi}^{A}\left(U_{A}^{\phi}\right)^{-1} d \theta
$$

\section{The Measure on the Fibres in $\mathscr{A} / \mathscr{G}_{m}$}

Referring to the discussion in Sect. 1 , the restriction of the measure $\mu$ to the fiber $\xi^{-1}(\gamma)$ should be the integral in $\mathscr{A}$ over $(\xi \circ \rho)^{-1}(\gamma)$, up to a factor of the volume of $\mathscr{G}_{m}$. Formally,

$$
\text { volume } \mathscr{G}_{m} \times \int_{\xi^{-1}(\gamma)} f([A]) \mu_{\text {fiber }}=\int_{\left(\xi_{\circ} \rho\right)^{-1}(\gamma)} f(A) e^{-S(A)} \mathscr{D} A
$$

We will evaluate the right-hand side as an integral over $\operatorname{ker} P_{\theta} \times \mathscr{G}_{m}$ of something which is constant in the $\mathscr{G}_{m}$ direction. Formally dividing by the volume of $\mathscr{G}_{m}$, we will obtain

\section{Theorem 3.1.}

$$
\int_{\xi^{-1}(\gamma)} f([A]) \mu_{\text {fiber }}=\operatorname{det}^{1 / 2}\left(D_{A}^{*} P_{\theta} D_{A}\right) \int_{\text {ker } P_{\theta}} f(A+\tau) e^{-S(A+\tau)} \mathscr{D} \tau,
$$

where $A$ is any connection in $(\xi \circ \rho)^{-1}(\gamma)$.

Figure 3.1 indicates the relation between $\gamma, \xi^{-1}(\gamma)$, and $(\xi \circ \rho)^{-1}(\gamma)$. 


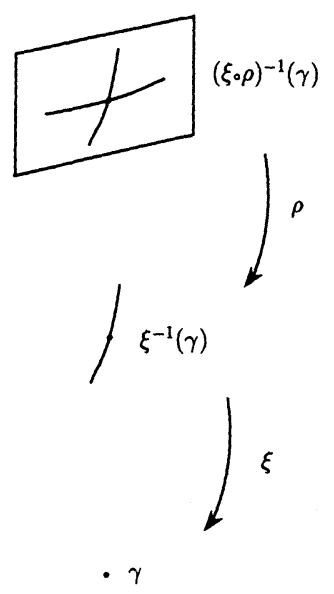

Fig. 3.1

Let $A$ be any connection in $\left(\xi_{\circ}^{\circ} \rho\right)^{-1}(\gamma)$. As shown in Sect. 2, every other such connection is of the form $\psi \cdot(A+\tau)$, where $\psi \in \mathscr{G}_{m}$ and $\tau \in \operatorname{ker} P_{\theta}$. The connection $A$ represents a choice of origin in the affine fiber.

We intend to express the integral over $\xi^{-1}(\gamma)$ as an integral over $\operatorname{ker} P_{\theta} \times \mathscr{G}_{m}$. The change of variables $J$ between these two spaces is

$$
\psi \cdot(A+\tau)=J(\tau, \psi)
$$

Thus,

$$
\left.\mathscr{D} A\right|_{(\zeta \circ \rho)^{-1}(\gamma)}=\operatorname{det}^{1 / 2}(\mathrm{~d} J * \mathrm{~d} J) \operatorname{vol}_{\mathrm{ker} P_{\theta}} \operatorname{vol}_{\mathscr{G}_{m}},
$$

and

$$
\mu_{\text {fiber }}=\operatorname{det}^{1 / 2}(\mathbf{d} J * \mathbf{d} J) \operatorname{vol}_{\mathrm{ker} P_{\theta}} .
$$

The differential $\mathbf{d} J$ maps $T_{(\tau, \psi)}\left(\operatorname{ker} P_{\theta} \times \mathscr{G}_{m}\right)$ to $T_{\psi \cdot(A+\tau)}\left(\left(\xi^{\circ} \rho\right)^{-1}(\gamma)\right)$. Since $\operatorname{ker} P_{\theta}$ is linear,

$$
T_{(\tau, \psi)}\left(\operatorname{ker} P_{\theta} \times \mathscr{G}_{m}\right) \sim \operatorname{ker} P_{\theta} \oplus \wedge^{0}(M, \mathbf{g}) .
$$

To evaluate the Jacobian, we prove a similar decomposition for the tangent space to $(\xi \circ \rho)^{-1}(\gamma)$; namely,

$$
T_{\psi \cdot(A+\tau)}\left((\xi \circ \rho)^{-1}(\gamma)\right) \sim \operatorname{ad}_{\psi}\left(\operatorname{ker} P_{\theta}\right) \oplus P_{\theta} D_{\psi \cdot(A+\tau)}\left(\wedge^{0}(M, \mathrm{~g})\right) .
$$

The tangent space in $\mathscr{A}$ to $(\xi \circ \rho)^{-1}(\gamma)$ at the point $\psi \cdot(A+\tau)$ consists of the elements obtained by separate variations of $\tau$ and $\psi$. These are

$$
\begin{aligned}
\left.\frac{d}{d t}\right|_{t=0} \psi \cdot(A+\tau+t \delta \tau) & =\psi \cdot(A+\tau)+\left.\frac{d}{d t}\right|_{t=0} t \operatorname{ad}_{\psi^{-1}} \delta \tau \\
& =\operatorname{ad}_{\psi^{-1}} \delta \tau,
\end{aligned}
$$


and

$$
\begin{aligned}
\left.\frac{d}{d t}\right|_{t=0} \psi e^{t f} \cdot(A+\tau) & =\left.\frac{d}{d t}\right|_{t=0} e^{t f} \cdot(\psi \cdot(A+\tau)) \\
& =D_{\psi \cdot(A+\tau)} f,
\end{aligned}
$$

for $f \in \wedge^{0}(M, \mathbf{g})$. Thus, the spaces $\operatorname{ad}_{\psi^{-1}}\left(\operatorname{ker} P_{\theta}\right)$ and $D_{\psi \cdot(\boldsymbol{A}+\tau)}\left(\wedge^{0}(M, \mathbf{g})\right)$ span $T_{\psi \cdot(A+\tau)}\left((\xi \circ \rho)^{-1}(\gamma)\right)$. These spaces are linearly independent. Were they not, there would be some $A, \tau$ and $f$ as above for which $D_{A} f=\tau$. Since $f$ must be zero at the north pole, such an $f$ must vanish identically. Therefore, the direct sum of $\operatorname{ad}_{\psi^{-1}}\left(\operatorname{ker} P_{\theta}\right)$ with the projection of $D_{\psi \cdot(A+\tau)}\left(\wedge^{0}(M, g)\right)$ to the orthogonal complement of the first space is an orthogonal decomposition of $T_{(\xi \circ \rho)^{-1}(\gamma)} \mathscr{A}$. The projection $P_{\theta}$ is projection to the orthogonal complement of $\operatorname{ker} \boldsymbol{P}_{\theta}$. This commutes with $\operatorname{ad}_{\psi^{-1}}$, so $P_{\theta} D_{\psi \cdot(A+\tau)}\left(\wedge^{0}(M, \mathbf{g})\right)$ is the desired orthogonal complement of $\operatorname{ad}_{\psi^{-1}}\left(\operatorname{ker} P_{\theta}\right)$ in the tangent to $(\xi \circ \rho)^{-1}(\gamma)$, which completes the proof of the decomposition (3.1).

In terms of the above decompositions of the tangent spaces, the differential of $J$ is the operator-valued matrix,

$$
\mathbf{d} J=\left(\begin{array}{cc}
\operatorname{ad}_{\psi^{-1}} & 0 \\
0 & P_{\theta} D_{\psi \cdot(A+\tau)}
\end{array}\right),
$$

and

$$
\mathbf{d} J^{*} \mathrm{~d} J=\left(\begin{array}{cc}
\operatorname{ad}_{\psi-1}^{*} \operatorname{ad}_{\psi-1} & 0 \\
0 & D_{\psi \cdot(A+\tau)}^{*} P_{\theta} D_{\psi \cdot(A+\tau)}
\end{array}\right) .
$$

This reduces, due to the $G$-invariance of the metric and the usual formula for the effect of a gauge transformation on the covariant derivative, to

$$
\mathbf{d} J^{*} \mathbf{d} J=\left(\begin{array}{cc}
1 & 0 \\
0 & \operatorname{ad}_{\psi^{-1}} D_{A}^{*} P_{\theta} D_{A} \mathrm{ad}_{\psi}
\end{array}\right) .
$$

Thus, the Jacobian is

$$
\operatorname{det}^{1 / 2}\left(\mathbf{d} J^{*} \mathbf{d} J\right)=\operatorname{det}^{1 / 2}\left(\operatorname{ad}_{\psi^{-1}} D_{A}^{*} P_{\theta} D_{A} \operatorname{ad}_{\psi}\right)=\operatorname{det}^{1 / 2}\left(D_{A}^{*} P_{\theta} D_{A}\right)
$$

assuming that $\operatorname{det}\left[(\beta \alpha)^{*}(\beta \alpha)\right]=\operatorname{det}\left[\left(\alpha^{*} \alpha\right)\left(\beta^{*} \beta\right)\right]$ as in finite dimensions. This does not depend on $\psi$ or $\tau$, so the Jacobian is a constant along the fiber in $\mathscr{A} / \mathscr{G}_{m}$. This completes the derivation of Theorem 3.1 for the measure on the fiber.

To be able to compute the integral over the fiber, we now make a specific choice for the gauge-class of the origin in each fiber. This constitutes a section of $\mathscr{A} / \mathscr{G}_{m}$ over $\Omega G$. Since, for $\tau \in \operatorname{ker} P_{\theta},[\tau \wedge \tau]=0$,

$$
F_{A+\tau}=F_{A}+D_{A} \tau
$$

and the action is quadratic in $\tau$ :

$$
S(A+\tau)=\frac{1}{4}\left\langle F_{A}, F_{A}\right\rangle+\frac{1}{2}\left\langle P_{\phi} D_{A}^{*} F_{A}, \tau\right\rangle+\frac{1}{4}\left\langle\tau, P_{\phi} D_{A}^{*} D_{A} P_{\phi} \tau\right\rangle .
$$

The standard technique for integrating the exponential of a quadratic action is to shift the integration variable to a new variable, differing by a constant, in which the action is purely quadratic. For any shift $\tau^{\prime} \in \operatorname{ker} P_{\theta}$, the connection $\tilde{A}=A-\tau^{\prime}$ 
represents a new choice of origin in the fiber. The section we would like to use to compute the integral corresponds to a choice of origin for which $S(A+\tau)=S(\tilde{A}+\tilde{\tau})$ is purely quadratic in the shifted variable $\tilde{\tau}=\tau+\tau^{\prime}$. According to (3.3), for the linear term to vanish, $\tilde{A}$ must satisfy

$$
P_{\phi} D_{\tilde{A}}^{*} F_{\tilde{A}}=0
$$

Theorem 3.2. There exists a unique section of $\mathscr{A} / \mathscr{G}_{m}$ such that every point on this section has a representative $\tilde{A}$ satisfying $P_{\phi} D_{\tilde{A}}^{*} F_{\tilde{A}}=0$ and the continuity conditions in our statement of Stoke's Law 2.2.

To prove this, let $A$ be some connection in $(\xi \circ \rho)^{-1}(\gamma)$. Without loss of generality, we may assume $A$ satisfies the continuity condition. Were it not to do so, an appropriate gauge transformation would make the $\theta$-component continuous (even smooth) as a function of $\theta$. Adding an appropriately discontinuous element of ker $P_{\theta}$ (which we could choose to be piecewise covariant constant along longitudes) would allow the $\phi$-component to satisfy its part of the continuity condition. First we will show there is a unique $\tau^{\prime} \in \operatorname{ker} P_{\theta}$ such that $\tilde{A}=A-\tau^{\prime}$ satisfies Eq. (3.4) and the continuity condition. Then we will show the gauge class of this $\tilde{A}$ is independent of the initial choice for $A$.

In terms of the connection $A$, the condition that the shifted connection satisfy Eq. (3.4) is

$$
\left(P_{\phi} D_{A}^{*} D_{A} P_{\phi}\right) \tau^{\prime}=P_{\phi} D_{A}^{*} F_{A}
$$

Thus, the required $\tilde{A}$ will exist if the image of the operator $P_{\phi} D_{A}^{*} D_{A} P_{\phi}: \operatorname{ker} P_{\theta} \rightarrow \operatorname{ker} P_{\theta}$ contains $P_{\phi} D_{A}^{*} F_{A}$ for all $A \in \mathscr{A}$. A direct computation shows $P_{\phi} D_{A}^{*} F_{A}$ is orthogonal to every element of coker $\left(P_{\phi} D_{A}^{*} D_{A} P_{\phi}\right)$; hence, it lies in $\operatorname{im}\left(P_{\phi} D_{A}^{*} D_{A} P_{\phi}\right)$. This proves the existence of the required $\tilde{A}$.

The continuity condition uniquely determines $\tau^{\prime}$, and hence $\tilde{A}$, since $\operatorname{ker}\left(P_{\phi} D_{A}^{*} D_{A} P_{\phi}\right)$ is trivial on elements of $\operatorname{ker} P_{\theta}$ which are continuous along longitudes and vanish at the north and south poles. (Note that $\operatorname{ker}\left(P_{\phi} D_{A}^{*} D_{A} P_{\phi}\right)$ includes covariantly linear elements of $\operatorname{ker} P_{\theta}$.) Shifting $A$ by this $\tau^{\prime}$ defines the connection $\tilde{A} \in(\xi \circ \rho)^{-1}(\gamma)$ satisfying Eq. (3.4).

To show the gauge class of $\tilde{A}$ is independent of the initial choice $A$, we again note that any other such choice is of the form $A_{1}=\psi \cdot\left(A+\tau_{1}\right)$ for some $\psi \in \mathscr{G}_{m}$ and $\tau_{1} \in \operatorname{ker} P_{\theta}$ such that $A_{1}$ satisfies the continuity condition. In particular, $\tau_{1}$ must vanish at the north and south poles. This follows from the effect of a gauge transformation on the connection: $P_{\phi} A_{1}=\left(\psi^{-1} A_{\phi} \psi+\phi^{-1} \partial_{\phi} \psi\right) \mathrm{d} \phi+\tau_{1}$. The continuity of $\psi$ implies $\partial_{\phi} \psi$ must vanish at the north and south poles. By hypothesis, both $P_{\phi} A$ and $P_{\phi} A_{1}$ vanish there, so $\tau_{1}$ must follow suit. The usual formulas for the effect of a gauge transformation on the covariant derivative and the curvature imply that $\tau_{1}^{\prime}=\psi^{-1}\left(\tau^{\prime}+\tau_{1}\right) \psi$ satisfies Eq. (3.5) with $A_{1}$ replacing $A$. The vanishing of $\tau_{1}$ at the north and south poles implies this is the unique shift of $A_{1}$ to a connection which satisfies the continuity condition. This shifted connection is

$$
\begin{aligned}
A_{1}-\tau_{1}^{\prime} & =\psi \cdot\left(A+\tau_{1}\right)-\psi^{-1}\left(\tau^{\prime}+\tau_{1}\right) \psi \\
& =\psi \cdot A+\psi^{-1} \tau_{1} \psi-\psi^{-1} \cdot\left(\tau^{\prime}+\tau_{1}\right) \psi \\
& =\psi \cdot\left(A-\tau^{\prime}\right) .
\end{aligned}
$$


The condition (3.4), with the continuity requirement, thus distinguishes a welldefined choice of origin in the fiber of $\mathscr{A} / \mathscr{G}_{m}$ over each loop $\gamma$.

We now use this choice to compute the integral over the fiber. The purely quadratic action is

$$
S(\tilde{A}+\tilde{\tau})=\frac{1}{4}\left\langle F_{\tilde{A}}, F_{\tilde{A}}\right\rangle+\frac{1}{4}\left\langle D_{\tilde{A}} \tilde{\tau}, A_{\tilde{A}} \tilde{\tau}\right\rangle=S_{\gamma}+\frac{1}{4}\left\langle\tilde{\tau}, P_{\phi} D_{\tilde{A}}^{*} D_{\tilde{A}} P_{\phi} \tilde{\tau}\right\rangle,
$$

where, because $\tilde{A}$ depends only on $\gamma, S_{\gamma}$ is an action on $\Omega G$. The integral over the fiber is, from Theorem 3.1,

$$
\int_{(\xi \circ \rho)^{-1}(\gamma)} f([A]) \mu_{\mathrm{fiber}}=\operatorname{det}^{1 / 2}\left(D_{A}^{*} P_{\theta} D_{A}\right) e^{-S_{\gamma}} \int_{\operatorname{ker} P_{\theta}} f(\tilde{A}+\tilde{\tau}) e^{-(1 / 4)\left\langle\tilde{\tau}, P_{\phi} D_{A}^{*} D_{A} P_{\phi} \tilde{\tau}\right\rangle} \mathscr{D} \tilde{\tau} .
$$

For $f=1$, performing this Gaussian integral yields the push-forward measure $\xi_{*}(\mu)$ on $\Omega G$. For any $f$ independent of $\tilde{\tau}$, the integral over $\operatorname{ker} P_{\theta}$ is $\left(\operatorname{det}^{\prime}\right)^{-1 / 2}\left(P_{\phi} D_{A}^{*} D_{A} P_{\phi}\right)$, where the prime indicates the restriction to the orthogonal complement of the kernel of $P_{\phi} D_{A}^{*} D_{A} P_{\phi}$. Thus,

$$
\xi_{*}(\mu)=e^{-S_{\gamma}} \frac{\operatorname{det}^{1 / 2}\left(D_{A}^{*} P_{\theta} D_{A}\right)}{\left(\operatorname{det}^{\prime}\right)^{1 / 2}\left(P_{\phi} D_{A}^{*} D_{A} P_{\phi}\right)} \operatorname{vol}_{\Omega G},
$$

where $\operatorname{vol}_{\Omega G}$ indicates the measure on $\Omega G$ which the metric $\langle$,$\rangle on \mathscr{A}$ induces. More generally, if $f$ is a polynomial in $\tilde{\tau}$, the integral over $\operatorname{ker} P_{\theta}$ is some function of $[\tilde{A}]$ times the same determinant. We write this as $\hat{f}(\gamma)\left(\operatorname{det}^{\prime}\right)^{-1 / 2}\left(P_{\phi} D_{A}^{*} D_{A} P_{\phi}\right)$. For the integral over the fiber, we have shown

\section{Corollary 3.1.}

$$
\int_{\left(\xi_{\circ} \rho\right)^{-1}(\gamma)} f([A]) \mu_{\text {fiber }}=\hat{f}(\gamma) e^{-S_{\gamma}} \frac{\operatorname{det}^{1 / 2}\left(D_{A}^{*} P_{\theta} D_{A}\right)}{\left(\operatorname{det}^{\prime}\right)^{1 / 2}\left(P_{\phi} D_{A}^{*} D_{A} P_{\phi}\right)} .
$$

In particular,

$$
\xi_{*}(\mu)=e^{-s_{\gamma}} \frac{\operatorname{det}^{1 / 2}\left(D_{A}^{*} P_{\theta} D_{A}\right)}{\left(\operatorname{det}^{\prime}\right)^{1 / 2}\left(P_{\phi} D_{A}^{*} D_{A} P_{\phi}\right)} \operatorname{vol}_{\Omega G} .
$$

The most direct approach to computing the path integral for the expectation would have been to decompose each connection as $A=P_{\theta} A+P_{\phi} A=A_{\theta} \mathrm{d} \theta+A_{\phi} \mathrm{d} \phi$, and to integrate first over $A_{\phi}$, then over $A_{\theta}$. After a suitable shift of $A_{\phi}$, the first integration becomes a Gaussian, and so forth. This corresponds exactly to the above approach with $P_{\theta} A$ as the initial choice to represent the origin in the fiber. However, this approach tends to obscure the gauge-invariance of the distinguished choice of origin, thereby confusing the account of integration over the gauge orbits.

\section{The Integration over $\Omega G$}

In this section, we will evaluate $S_{\gamma}$, the ratio of determinants, and $\operatorname{vol}_{\Omega G}$. Letting $\mathscr{D} \gamma$ denote the measure on $\Omega G$ corresponding to the metric $\|\delta \gamma\|^{2}=$ $\int_{S^{1}}(\delta \gamma(\phi), \delta \gamma(\phi)) d \phi$, we will prove 


\section{Theorem 4.1.}

$$
\int_{(\xi \circ \rho))^{-1}(\gamma)} f([A]) \mu_{\text {fiber }}=\widehat{f} e^{-(1 / 8)\|\dot{\gamma}\|^{2}},
$$

where $\hat{f}$ is defined as in Corollary 3.1. In particular, up to a factor independent of $\gamma$,

$$
\xi_{*}(\mu)=e^{-(1 / 8)\|\dot{\gamma}\|^{2}} \mathscr{D} \gamma .
$$

The expectation of a gauge-invariant function is a normalization constant times the integral with respect to $\mu$. The above theorem thus implies

Corollary 4.1 .

$$
\langle f\rangle=\frac{1}{Z_{0}} \int_{\Omega G} \hat{f}(\gamma) e^{-(1 / 8)\|\dot{\gamma}\|^{2}} \mathscr{D} \gamma .
$$

4.1. Evaluating $S_{\gamma}$. We begin by showing that the induced action on $\Omega G$ is the energy action. That is,

$$
S_{\gamma}=\frac{1}{8}\|\dot{\gamma}\|^{2} \text {. }
$$

To prove this, we use the special property Eq. (3.4), of the section to compute

$$
S_{\gamma}(\tilde{A})=\frac{1}{4}\left\|F_{\tilde{A}}\right\|^{2} \text {. }
$$

In terms of the Hodge star operator, $D_{\tilde{A}}^{*}=* D_{\tilde{A}} *$. On Lie-algebra-valued 1-forms, $P_{\phi} *=* P_{\theta}$, so Eq. (3.4) is the condition

$$
P_{\theta} D_{\tilde{A}} * F=0 \text {. }
$$

That is, $* F$ must be covariantly constant along longitudes. Taking the standard metric on $S^{2}$, there must be some Lie-algebra-valued function $\alpha(\phi)$, for which

$$
F_{\theta \phi}=U_{\tilde{A}}^{-1} \alpha U_{\tilde{A}} \sin \theta \text {. }
$$

To determine $\alpha$, multiply both sides by $U_{\tilde{A}}$ on the left and $U_{\tilde{A}}^{-1}$ on the right, and then integrate over $\theta \in[0, \pi]$ :

$$
\int_{0}^{\pi} U_{\tilde{A}} F_{\theta \phi}^{\tilde{A}} U_{\tilde{A}}^{-1} d \theta=\alpha \int_{0}^{\pi} \sin \theta d \theta
$$

Comparison with Corollary 2.1 for $\gamma_{\tilde{A}}^{-1} \dot{\gamma}_{\tilde{A}}$ gives

$$
\alpha=\frac{1}{2} \gamma_{\tilde{A}}^{-1} \dot{\gamma}_{\tilde{A}} \text {. }
$$

Since the metric on $\Omega G$ is $G$-invariant, this gives

$$
\begin{aligned}
\left\|\boldsymbol{F}_{\tilde{A}}\right\|^{2} & =\int_{\boldsymbol{S}^{2}}\left(\boldsymbol{F}_{\theta \phi}^{\tilde{A}}, F_{\theta \phi}^{\tilde{A}}\right) \frac{1}{\sin ^{2} \theta} \sin \theta d \theta d \phi \\
& =\frac{1}{4} \int_{S^{2}}\left(\gamma_{\tilde{\tilde{A}}}^{-1} \dot{\gamma}_{\tilde{A}}(\phi), \gamma_{\tilde{\tilde{A}}}^{-1} \dot{\gamma}_{\tilde{A}}(\phi)\right) \sin \theta d \theta d \phi \\
& =\frac{1}{2} \int_{S^{1}}\left(\dot{\gamma}_{\tilde{A}}, \dot{\gamma}_{\tilde{A}}\right) d \phi \\
& =\frac{1}{2}\left\|\dot{\gamma}_{\tilde{A}}\right\|^{2},
\end{aligned}
$$

as Eq. (4.1) requires. 
4.2 The Ratio of Determinants. The ratio of determinants in Lemma 3.1 also reduces to a function of the loop $\gamma_{A}$; namely, a constant function. This is because the operators in numerator and denominator, though operating on different spaces, are closely related. The first is $D_{A} P_{\theta} D_{A}$ on $f \in \wedge^{0}(M, \mathrm{~g})$ for which $f(\theta=0)=0$. The second is $P_{\phi} D_{A}^{*} D_{A} P_{\phi}$ on the orthogonal complement in $\operatorname{ker} P_{\theta}$ of $\operatorname{ker}\left(P_{\phi} D_{A}^{*} D_{A} P_{\phi}\right)$. Suppose $\left\{f_{i}\right\}$ is a complete set of eigenfunctions of the first operator, so $D_{A}^{*} P_{\theta} D_{A} f_{i}=\lambda_{i} f_{i}$ for the corresponding eigenvalues $\left\{\lambda_{i}\right\}$. We will show that $P_{\phi} * D_{A}: \wedge^{0}(M, \mathbf{g}) \rightarrow \operatorname{ker} P_{\theta}$ has trivial kernel on the span of $\left\{f_{i}\right\}$ and that its cokernel is contained in the kernel of $P_{\phi} D_{A}^{*} D_{A} P_{\phi}$. It thus maps $\left\{f_{i}\right\}$ one-to-one to a complete set in the orthogonal complement of the latter kernel. Moreover, $P_{\phi} * D_{A}$ takes eigenfunctions of the first operator to eigenfunctions of the second, preserving the eigenvalue. That is, apart from zero eigenvalues, the two operators have the same spectrum. We will thus conclude,

$$
\operatorname{det}\left(D_{A}^{*} P_{\theta} D_{A}\right)=\operatorname{det}^{\prime}\left(P_{\phi} D_{A}^{*} D_{A} P_{\phi}\right) \text {. }
$$

To see that $P_{\phi} * D_{A}$ has trivial kernel, suppose $P_{\phi} * D_{A} f=0$. As above, this implies $P_{\theta} D_{A} f=0$. The only solution satisfying $f(\theta=0)=0$ is the trivial solution.

If $\eta$ is in the cokernel of $P_{\phi} * D_{A} f$, then

$$
0=\left\langle\eta, P_{\phi} * D_{A} f\right\rangle=\int_{S^{2}} P_{\phi} \eta \wedge D_{A} f
$$

since $*^{2}$ is 1 on 1 -forms. Integration by parts shows that, for this to hold for arbitrary $f, D_{A} P_{\phi} \eta$ must vanish, so $\eta \in \operatorname{ker}\left(P_{\phi} D_{A}^{*} D_{A} P_{\phi}\right)$. This shows $\operatorname{coker}\left(P_{\phi} * D_{A}\right) \subset$ $\operatorname{ker}\left(P_{\phi} D_{A}^{*} D_{A} P_{\phi}\right)$.

The spectra are identical, since

$$
\begin{aligned}
P_{\phi} D_{A}^{*} D_{A} P_{\phi}\left(P_{\phi} * D_{A} f_{i}\right) & =P_{\phi} * D_{A} * D_{A} P_{\phi}\left(P_{\phi} * D_{A} f_{i}\right) \\
& =P_{\phi} * D_{A} D_{A}^{*} P_{\theta} D_{A} f_{i} \\
& =\lambda_{i}\left(P_{\phi} * D_{A} f_{i}\right) .
\end{aligned}
$$

This proves the validity of Eq. (4.2), which implies the ratio of determinants in Corollary 3.1 is the constant 1 .

4.3 Another Jacobian. The measure $\mu$ is the product of the measure $\mu_{\mathrm{fiber}}$ and the measure $\operatorname{vol}_{\Omega G}$, where the metric in $\mathscr{A}$ on $T_{\tilde{A}}^{H}\left(\mathscr{A} / \mathscr{G}_{m}\right)$, the orthogonal complement of the fiber directions at each $\tilde{A}$, defines the induced metric corresponding to $\operatorname{vol}_{\Omega G}$. The map $\xi$ acts as a change of variables from $\mathscr{A} / \mathscr{G}_{m}$ to $\Omega G$. The tangent space to $\mathscr{A}$ decomposes into the tangent in directions which project under $\rho_{*}$ to the fiber in $\mathscr{A} / \mathscr{G}_{m}$ plus the orthogonal complement of these directions. The differential $\rho_{*}$ of the projection maps this orthogonal complement to $T^{H}\left(\mathscr{A} / \mathscr{G}_{m}\right)$ and induces the metric on the latter space. On the tangent spaces the change of variables is

$$
\left.\mathrm{d} \xi\right|_{H}: T^{H}\left(\mathscr{A} / \mathscr{G}_{m}\right) \rightarrow T \Omega G \sim \Omega \mathrm{g} .
$$

Thus, $\operatorname{vol}_{\Omega G}$ is $\mathscr{D} \gamma$ times the Jacobian $\operatorname{det}^{-1 / 2}\left(\left.\left.\mathbf{d} \xi\right|_{H} ^{*} \mathbf{d} \xi\right|_{H}\right)$ of this mapping. (The minus sign in the exponent is consistent with the plus sign in the exponent in the derivation of Theorem 3.1, since in that case the map $J$ went in the opposite direction.)

We will show the Jacobian $\operatorname{det}\left(\left.\left.\mathbf{d} \xi\right|_{H} ^{*} \mathbf{d} \xi\right|_{H}\right)$ is independent of $\gamma$. First compute 
$\left.\mathbf{d} \xi\right|_{H}$ on $\eta \in T \mathscr{A}$ representing an element of $T^{H}\left(\mathscr{A} / \mathscr{G}_{m}\right)$. Since $\rho_{*} \eta \in T^{H}\left(\mathscr{A} / \mathscr{G}_{m}\right), \eta$ must be orthogonal to the fiber directions, which means

$$
\left\langle P_{\theta} D_{A} f, \eta\right\rangle=0 \text { and } \eta=P_{\theta} \eta,
$$

for all continuous 0 -forms $f$ with $f(\theta=0)=0$. To keep track of a possible discontinuity at the north pole, which will prove important, regard $S^{2}$ as a disk $D$ in the plane with the boundary circle $\partial D$ identified to a point. Take this point to correspond to the north pole, the origin to correspond to the south pole, and radial lines to correspond to longitudes. The condition on $\eta$ becomes, upon integrating by parts,

$$
-\int_{D} f \wedge D_{A} P_{\phi} * \eta+\int_{\partial D} f \wedge P_{\phi} * \eta=0
$$

For this to hold for all allowed $f, \eta$ must satisfy

$$
D_{A} P_{\phi} * \eta=0 \text { and } \int_{\partial D} P_{\phi} * \eta=0
$$

since continuity requires that $f$ be constant on $\partial D$. With the standard metric on $S^{2}$, the solution to the first condition is

$$
\eta_{\theta}=\frac{1}{\sin \theta} U_{A}^{-1} \bar{\eta}(\phi) U_{A},
$$

where $\bar{\eta}$ could be any map from $S^{1}$ to $\mathrm{g}$. The second condition restricts $\bar{\eta}$, requiring

$$
\int_{0}^{2 \pi} U_{A}^{\phi}(\pi)^{-1} \bar{\eta} U_{A}^{\phi}(\pi) d \phi=0
$$

The map $\left.\mathbf{d} \xi\right|_{H}$ from the tangent $\eta$ to a tangent to $\Omega G$ at $\gamma_{A}$ follows from the effect of $\eta$ on parallel transport:

$$
\begin{aligned}
\left.\mathbf{d} \xi\right|_{H}(\eta) & =\left.\frac{d}{d t}\right|_{t=0}(\rho \circ \xi)(A+t \eta) \\
& =\left.\frac{d}{d t}\right|_{t=0} U_{A+t \eta}^{0}(\pi) U_{A+t \eta}^{\phi}(\pi)^{-1} .
\end{aligned}
$$

To first order in $t$,

$$
U_{A+t \eta} \sim U_{A}\left[1+t U_{A}^{-1}\left(\int_{0}^{\theta} U_{A} \eta_{\theta} U_{A}^{-1} d \bar{\theta}\right) U_{A}\right],
$$

as follows directly from the parallel transport equation (0.1). For $\eta$ satisfying (4.3), this means, abbreviating $\xi(A)$ as $\gamma_{A}$,

$$
\gamma_{A+t \eta} \sim \gamma_{A}\left\{1+t C\left[\gamma_{A}^{-1} \bar{\eta}(0) \gamma_{A}-\bar{\eta}(\phi)\right]\right\},
$$

where $C$ is an infinite constant,

$$
C=\int_{0}^{\pi} \frac{1}{\sin \theta} d \theta .
$$

Thus, the change of variable is,

$$
\left.\mathbf{d} \xi\right|_{H}(\eta)=C\left[\gamma_{A}^{-1} \bar{\eta}(0) \gamma_{A}-\bar{\eta}(\phi)\right] .
$$


To compute the determinant of $\left.\left.\mathbf{d} \xi\right|_{H} ^{*} \mathbf{d} \xi\right|_{H}$, suppose $\left\{\eta^{i}\right\}$ is an orthonormal basis for the orthogonal tangent space. Since

$$
\begin{aligned}
\delta^{i j}=\left\langle\eta^{i}, \eta^{j}\right\rangle & =\int_{0}^{2 \pi} \int_{0}^{\pi}\left(U_{A}^{-1} \bar{\eta}^{i} U_{A}, U_{A}^{-1} \bar{\eta}^{j} U_{A}\right) \frac{1}{\sin \theta} d \theta d \phi \\
& =C \int_{0}^{2 \pi}\left(\bar{\eta}^{i}, \bar{\eta}^{j}\right) d \phi,
\end{aligned}
$$

the corresponding $\bar{\eta}^{i}$ are orthogonal with norm $\sqrt{1 / C}$ as elements of $\Omega(\mathrm{g})$. In the given basis, the $i j$-th element of the matrix for $\left.\left.\mathbf{d} \xi\right|_{H} ^{*} \mathbf{d} \xi\right|_{H}$ is

$$
\begin{aligned}
& \left\langle\left.\mathbf{d} \xi\right|_{H}\left(\eta^{i}\right),\left.\mathbf{d} \xi\right|_{H}\left(\eta^{j}\right)\right\rangle \\
& =C^{2} \int_{0}^{2 \pi}\left(\left[\gamma_{A}^{-1} \bar{\eta}^{i}(0) \gamma_{A}-\bar{\eta}^{i}(\phi)\right],\left[\gamma_{A}^{-1} \bar{\eta}^{j}(0) \gamma_{A}-\bar{\eta}^{j}(\phi)\right]\right) d \phi \\
& \quad=C^{2}\left\{2 \pi\left(\bar{\eta}^{i}(0), \bar{\eta}^{j}(0)\right)-\left[\int_{0}^{2 \pi}\left(\gamma_{A}^{-1} \bar{\eta}^{i}(0) \gamma_{A}, \bar{\eta}^{j}(\phi)\right) d \phi+(i \leftrightarrow j)\right]+\int_{0}^{2 \pi}\left(\bar{\eta}^{i}, \bar{\eta}^{j}\right)\right\} .
\end{aligned}
$$

As in (4.5) the last term is $\sqrt{1 / C} \delta^{i j}$. Writing $\gamma_{A}$ as $U_{A}^{0}(\pi) U_{A}^{\phi}(\pi)^{-1}$ and using the $G$-invariance of the inner product changes the cross term to

$$
-\left[\left(U_{A}^{0}(\pi)^{-1} \bar{\eta}^{i}(0) U_{A}^{0}(\pi), \int_{0}^{2 \pi} U_{A}^{\phi}(\pi)^{-1} \bar{\eta}^{j}(\phi) U_{A}^{\phi}(\pi) d \phi\right)+(i \leftrightarrow j)\right] .
$$

By the boundary condition (4.4) on the $\vec{\eta}$ 's, each of these summands vanishes. Thus the matrix for $\left.\left.\mathbf{d} \xi\right|_{H} ^{*} \mathbf{d} \xi\right|_{H}$ is the same as the matrix for

$$
C\left[1+2 \pi C(\text { evaluation at } 0)^{*}(\text { evaluation at } 0)\right] \text {. }
$$

This does not depend on the loop $\gamma_{A}$, so the determinant is some universal constant, as claimed. Hence, up to this constant, integration with respect to $\operatorname{vol}_{\Omega G}$ is integration with respect to $\mathscr{D} \gamma$.

Replacing each term in Corollary 3.1 with its corresponding expression in terms of loops completes the proof of Theorem 4.1. Corollary 4.1 follows from the observation that the integral with respect to $\mu$ is the integral over each fiber with respect to $\mu_{\text {fiber }}$ followed by integration over $\Omega G$ with respect to vol $\mathrm{v}_{\Omega G}$. The above constant, representing the switch from vol $\operatorname{sG}_{\text {to }} \mathscr{D} \gamma$, simply becomes an extra factor in the normalization constant $Z_{0}$.

\section{Some Examples}

We begin this section by computing the expectations of functions of the form

$$
f=f^{1}\left(\gamma\left(\phi_{1}\right)\right) f^{2}\left(\gamma\left(\phi_{2}\right)\right) \cdots f^{n}\left(\gamma\left(\phi_{n}\right)\right),
$$

where $f^{i}: G \rightarrow \mathbf{R}$. As a function on $\mathscr{A}, \gamma\left(\phi_{i}\right)$ is the Wilson loop determined by the closed path which runs from the north pole to the south pole along the longitude at $\phi=0$ and back along the longitude at $\phi_{i}$. As shown in Sect. 2, $\gamma\left(\phi_{i}\right)$ is gauge-invariant, so $f$ is a gauge-invariant function of the connection.

The expectation of $f$ is a normalization factor times its integral in the Wiener 
measure. To compute this integral, we refer to Glimm and Jaffe [8, Chap. 3, Sect. 1] for a formula relating the (conditional) Wiener measure to the heat kernel. Regarding $\Omega G$ as paths in $G$ which begin and end at the identity and applying this formula to paths in $G$ gives,

$$
\begin{aligned}
& \left\langle f^{1}\left(\gamma\left(\phi_{1}\right)\right) \cdots f^{n}\left(\gamma\left(\phi_{n}\right)\right)\right\rangle \\
& \quad=\frac{1}{Z_{0}} \int_{\Omega G} f^{1}\left(\gamma\left(\phi_{1}\right)\right) \cdots f^{n}\left(\gamma\left(\phi_{n}\right)\right) e^{-(1 / 8)\|\dot{\gamma}\|^{2}} \mathscr{D} \gamma \\
& \quad=\frac{1}{Z_{0}} \operatorname{kernel}\left(e^{-(1 / 2) \phi_{1} \Delta} f^{1} e^{(1 / 2)\left(\phi_{2}-\phi_{1}\right) \Delta} f^{2} \cdots f^{n} e^{-(1 / 2)\left(2 \pi-\phi_{n}\right) \Delta}\right)(1,1),
\end{aligned}
$$

where $\Delta$ is the $G$-invariant Laplacian on $G$, and the $f^{i}$ act by multiplication. In Glimm and Jaffe, the energy is $\frac{1}{2}\|\dot{\gamma}\|^{2}$ rather than $\frac{1}{8}\|\dot{\gamma}\|^{2}$. The difference in the measures is a constant factor, which we absorb into the normalization constant $Z_{0}$. Since $\Delta$ is $G$-invariant, we may write

$$
e^{-(1 / 2) \phi \Delta}\left(g_{1}, g_{2}\right)=H\left(g_{1}^{-1} g_{2} ; \phi\right) \text {. }
$$

The above formula is thus,

$$
\begin{aligned}
\left\langle f^{1}\left(\gamma\left(\phi_{1}\right)\right) \cdots f^{n}\left(\gamma\left(\phi_{n}\right)\right)\right\rangle= & \frac{1}{Z_{0}} \int_{G} \cdots \int_{G} H\left(g_{1} ; \phi_{1}\right) f^{1}\left(g_{1}\right) H\left(g_{1}^{-1} g_{2} ; \phi_{2}-\phi_{1}\right) \cdots \\
& \cdot f^{n}\left(g_{n}\right) H\left(g_{n}^{-1} ; 2 \pi-\phi_{n}\right) d g_{1} \cdots d g_{n} .
\end{aligned}
$$

We begin by computing the normalization constant $Z_{0}$. It is the Wiener measure of the function of the above form for which all the $f^{i}$ are the constant function equal to the real number 1 . Due to the multiplicative property of the heat kernel, which is

$$
\int_{G} H\left(g_{1}^{-1} g ; \phi_{1}\right) H\left(g^{-1} g_{2} ; \phi_{2}\right) d g=H\left(g_{1}^{-1} g_{2} ; \phi_{1}+\phi_{2}\right),
$$

the integral on the right-hand side of Eq. (5.1) collapses in this case to give

$$
Z_{0}=H(1 ; 2 \pi) \text {. }
$$

To compute the measure of more general functions, we refer to the expansion in Fegan [9] for the kernel in terms of the characters of the highest-weight representations of $G$. The character of a representation at an element $g \in G$ is the trace of the linear transformation corresponding to $g$ in that representation. The expansion we will use, which is a consequence of the fact that the characters are the eigenfunctions of the Laplacian, is

$$
H(g ; \phi)=\sum_{\lambda} \chi_{\lambda}(1) \chi_{\lambda}(g) e^{-2 c(\lambda) \phi} .
$$

The sum is over highest weights in a fixed Weyl chamber and $c(\lambda)$ is the eigenvalue of $-\Delta$ in the representation labelled by $\lambda$. (This differs slightly from the expression in Fegan, due to a difference in conventions for the sign of the Laplacian and its numerical coefficient in the heat equation. We follow Glimm and Jaffe who take the Laplacian to have negative eigenvalues and to appear with a factor of $\frac{1}{2}$ in the 
heat equation). The characters are normalized so that

$$
\chi_{\lambda}(1)=\text { the dimension of the representation indexed by } \lambda \text {. }
$$

In this normalization, Fegan also gives the convolution property

$$
\int \chi_{\lambda}(\bar{g}) \chi_{\mu}\left(\bar{g}^{-1} g\right) d \bar{g}=\delta_{\lambda \mu} \frac{\chi_{\lambda}(g)}{\chi_{\lambda}(\mathbf{1})} .
$$

To integrate functions against the heat kernel we will also expand them as sums of characters;

$$
f(g)=\sum f_{\mu} \chi_{\mu}(g)
$$

As usual for eigenfunction expansions, multiplying both sides by a character, integrating over $G$, and using the convolution property (which is an orthogonality relation) yields a formula for the coefficients. In this case,

$$
f_{\mu}=\int f(g) \chi_{\mu}\left(g^{-1}\right) d g .
$$

Substitution the expansions for $H$ and the $f^{i}$ into Eqs. (5.1) and (5.2) yields an expression for the expectation. We will compute this in the case $G=S U(2)$ to obtain

Corollary 5.1. For $G=S U(2)$,

$$
\begin{aligned}
& \left\langle f^{1}\left(\gamma\left(\phi_{1}\right)\right) \cdots f^{n}\left(\gamma\left(\phi_{n}\right)\right)\right\rangle \\
& =\frac{1}{\sum_{\rho} \chi_{\rho}(\mathbf{1}) \chi_{\rho}(\mathbf{1}) e^{-\rho(\rho+1) 2 \pi}} \sum_{\mu} f_{\mu_{1}}^{1} \cdots f_{\mu_{n}}^{n} \sum_{v} \chi_{\mu_{0}}(\mathbf{1}) \chi_{v_{n}}(\mathbf{1}) \\
& \quad \cdot \exp \left(-\sum_{i=0}^{n} v_{i}\left(v_{i}+1\right)\left(\phi_{i+1}-\phi_{i}\right)\right)
\end{aligned}
$$

where $\mu$ and $v$ are multi-indices: $\mu=\left(\mu_{0}, \mu_{1} \cdots \mu_{n}\right)$ and $v=\left(v_{1}, v_{2}, \ldots, v_{n}\right)$. For each $i, \mu_{i}$ ranges over all positive elements of $\mathbf{Z} / 2$, while $v_{i}$ starts at $\left|v_{i-1}-\mu_{i}\right|$ and increases by integer increments to $v_{i-1}+\mu_{i}$. Here, and in the exponential, $v_{0} \equiv \mu_{0}$. Finally, $\phi_{0} \equiv 0$ and $\phi_{n+1} \equiv 2 \pi$.

To prove this, we require the following lemma:

Lemma 5.1.

$$
\int_{S U(2)} \chi_{\lambda}(\bar{g}) f(\bar{g}) H\left(\bar{g}^{-1} g ; \phi\right) d \bar{g}=\sum_{\mu} f_{\mu} \sum_{v=|\lambda-\mu|}^{\lambda+\mu} \chi_{\nu}(g) e^{-v(v+1) \phi},
$$

where $\mu$ ranges through the positive half-integers, and the sum over $v$ is by integer increments.

To prove the lemma, we first substitute in the expansions for $f$ and $H$,

$$
\int_{S U(2)} \chi_{\lambda}(\bar{g}) f(\bar{g}) H\left(\bar{g}^{-1} g ; \phi\right) d \bar{g}=\sum_{\mu, \rho} \int \chi_{\lambda}(\bar{g}) f_{\mu} \chi_{\mu}(\bar{g}) \chi_{\rho}(\mathbf{1}) \chi_{\rho}\left(\bar{g}^{-1} g\right) d \bar{g} e^{-2 c(\rho) \phi} .
$$

Next, we again refer to Fegan for a few facts about $S U(2)$. Take the highest weights to be positive elements of $\mathbf{Z} / 2$. The eigenvalues of $-\Delta$ are then $c(\lambda)=\frac{1}{2} \lambda(\lambda+1)$. For future reference, we note $\chi_{\lambda}(1)=2 \lambda+1$.

To evaluate the integral, expand $\chi_{\lambda}(\bar{g}) \chi_{\mu}(\bar{g})$ as a sum of characters and use the 
convolution property. In $S U(2)$, the sum is the Clebsch-Gordon series

$$
\chi_{\lambda}(\bar{g}) \chi_{\mu}(\bar{g})=\sum_{\nu=|\lambda-\mu|}^{\lambda+\mu} \chi_{\nu}(\bar{g})
$$

where, again, the sum over $v$ is by integer increments. Making these substitutions reduces the integral to

$$
\begin{aligned}
\int_{S U(2)} \chi_{\lambda}(\bar{g}) f(\bar{g}) H\left(\bar{g}^{-1} g ; \phi\right) d \bar{g} & =\sum_{\mu, \rho} f_{\mu} \chi_{\rho}(\mathbf{1}) \sum_{\nu=|\lambda-\mu|}^{\lambda+\mu} \delta_{\mu \nu} \frac{\chi_{v}(g)}{\chi_{\nu}(\mathbf{1})} e^{-\rho(\rho+1) \phi} \\
& =\sum_{\mu} f_{\mu} \sum_{\nu=|\lambda-\mu|}^{\lambda+\mu} \chi_{\nu}(g) e^{-v(v+1) \phi},
\end{aligned}
$$

proving the lemma.

We now use the lemma to show by induction on $n$ that

$$
\begin{gathered}
\int_{S U(2)} \cdots \int_{S U(2)} H\left(g_{1} ; \phi\right) f^{1}\left(g_{1}\right) \cdots f^{n}\left(g_{n}\right) H\left(g_{n}^{-1} g_{n+1} ; \phi_{n+1}-\phi_{n}\right) d g_{1} \cdots d g_{n} \\
=\sum_{\mu} f_{\mu_{1}}^{1} \cdots f_{\mu_{n}}^{n} \sum_{v} \chi_{\mu_{0}}(1) \chi_{v_{n}}\left(g_{n+1}\right) \exp \left(-\sum_{i=1}^{n} v_{i}\left(v_{i}+1\right)\left(\phi_{i+1}-\phi_{i}\right)\right),
\end{gathered}
$$

where the sums over the $\mu_{i}$ are over elements of $\mathbf{Z} / 2$ greater than zero and the sums over the $v_{i}$ are by integer increments in $\mathbf{Z} / 2$. When $g_{n+1}=1$ and $\phi_{n+1}=2 \pi$, the above equation, with Eq. (5.2) for $Z_{0}$ and Eq. (5.1), is the corollary. Suppose, as the induction hypothesis, that Eq. (5.5) holds for some $n$. Abbreviating the right-hand side by $\sum E_{v_{n}} \chi_{v_{n}}\left(g_{n+1}\right)$, and using this hypothesis to evaluate the integral in the $(n+1)$-case, ${ }^{v_{n}}$

$$
\begin{gathered}
\int \cdots \int H\left(g_{1} ; \phi\right) f^{1}\left(g_{1}\right) \cdots f^{n+1}\left(g_{n+1}\right) H\left(g_{n+1}^{-1} g_{n+2} ; \phi_{n+2}-\phi_{n+1}\right) d g_{1} \cdots d g_{n} d g_{n+1} \\
=\int_{S U(2)} \sum_{v_{n}} E_{v_{n}} \chi_{v_{n}}\left(g_{n+1}\right) f^{n+1}\left(g_{n+1}\right) H\left(g_{n+1}^{-1} g_{n+2} ; \phi_{n+2}-\phi_{n+1}\right) d g_{n+1} .
\end{gathered}
$$

Lemma 5.1 computes the integral on the right-hand side as

$$
\sum_{v_{n}} E_{v_{n}} \sum_{\mu_{n+1}} f_{\mu_{n+1}}^{n+1} \sum_{v_{n+1}}^{v_{n}+\mu_{n n}-\mu_{n+1} \mid} \chi_{v_{n+1}}\left(g_{n+2}\right) e^{-v_{n+1}\left(v_{n+1}+1\right)\left(\phi_{n+2}-\phi_{n+1}\right)} .
$$

Substituting back for $E_{v_{n}}$ proves the $(n+1)$-case.

We now show Eq. (5.5) holds for $n=1$; that is,

$$
\begin{aligned}
& \int_{S U(2)} H\left(g_{1} ; \phi_{1}\right) f\left(g_{1}\right) H\left(g_{1}^{-1} g_{2} ; \phi_{2}-\phi_{1}\right) d g_{1} \\
& \quad=\sum_{\mu_{0}, \mu_{1}} f_{\mu_{1}} \sum_{\nu=\left|\mu_{0}-\mu_{1}\right|}^{\mu_{0}+\mu_{1}} \chi_{\mu_{0}}(1) \chi_{v}(g) e^{-\left[\mu_{0}\left(\mu_{0}+1\right) \phi_{1}+v(v+1)\left(\phi_{2}-\phi_{1}\right)\right]} .
\end{aligned}
$$

Substituting its expansion in characters for $H\left(g_{1} ; \phi_{1}\right)$ makes the left-hand side

$$
\sum_{\mu_{0}} \chi_{\mu_{0}}(1) \int_{S U(2)} \chi_{\mu_{0}}\left(g_{1}\right) f\left(g_{1}\right) H\left(g_{1}^{-1} g_{2} ; \phi_{2}-\phi_{1}\right) d g_{1} e^{-\mu_{0}\left(\mu_{0}+1\right) \phi_{1}} .
$$

Applying Lemma 5.1 once more yields Eq. (5.5) for the case $n=1$. This completes the proof by induction, thereby establishing Eq. (5.5) for all $n$. Corollary 5.1 follows immediately. For $G=S U(N)$, the same techniques would produce a similar result, but the analogue of the Clebsch-Gordon series is complicated. 
We now specialize further to the case in which $f$ depends on the value of $\gamma$ at only one point $\phi$. In particular, we take $f$ to be the trace of $\gamma(\phi)$ in the adjoint representation. That is, $f(g)=\chi_{1}(g)$. The expansion for $f$ is then trivial: $f_{\mu}=\delta_{\mu 1}$. Corollary 5.1 becomes

$$
\left\langle\chi_{1}(\gamma(\phi))\right\rangle=\frac{\sum_{\mu} \sum_{v=|\mu-1|}^{\mu+1} \chi_{\mu}(1) \chi_{v}(1) e^{-[\mu(\mu+1) \phi+v(v+1)(2 \pi-\phi)]}}{\sum_{\mu} \chi_{\mu}(1) \chi_{\mu}(1) e^{-\mu(\mu+1) 2 \pi}} .
$$

To close, we compute the expectation of a more general Wilson loop; namely, parallel transport from the north pole along the longitude at $\phi_{1}$ to the south pole, and back along the longitude at $\phi_{2}$. This Wilson loop is $\gamma\left(\phi_{1}\right)^{-1} \gamma\left(\phi_{2}\right)$. Notice that when $\phi_{1}=0$ this reduces to the previous example. Further, if the theory is invariant under rotations about the north pole, replacing $\phi$ by $\phi_{2}-\phi_{1}$ in the above formula should give the expectation for the corresponding function of this Wilson loop. In fact, for $G=S U(2)$, the expectation of the trace in any representation $\lambda$ of this loop is

\section{Corollary 5.2}

$$
\left\langle\chi_{\lambda}\left(\gamma\left(\phi_{1}\right)^{-1} \gamma\left(\phi_{2}\right)\right)\right\rangle=\frac{\sum_{\mu} \sum_{\nu=|\lambda-\mu|}^{\lambda+\mu} \chi_{\mu}(1) \chi_{\nu}(1) e^{-\mu(\mu+1)\left(\phi_{2}-\phi_{1}\right)} e^{-v(v+1)\left[2 \pi-\left(\phi_{2}-\phi_{1}\right)\right]}}{\sum_{\mu} \chi_{\mu}(1) \chi_{\mu}(1) e^{-\mu(\mu+1) 2 \pi}} .
$$

To prove this directly, we begin with the expression for the expectation,

$$
\begin{aligned}
& \left\langle\chi_{\lambda}\left(\gamma\left(\phi_{1}\right)^{-1} \gamma\left(\phi_{2}\right)\right)\right\rangle \\
& \quad=\int H\left(g_{1} ; \phi_{1}\right) \chi_{\lambda}\left(g_{1}^{-1} g_{2}\right) H\left(g_{1}^{-1} g_{2} ; \phi_{2}-\phi_{1}\right) H\left(g_{2}^{-1} ; 2 \pi-\phi_{2}\right) d g_{1} d g_{2} .
\end{aligned}
$$

First, we change variables in the $g_{2}$-integration to $g=g_{1}^{-1} g_{2}$, using the invariance of the volume: $d\left(g_{1}^{-1} g_{2}\right)=d g_{2}$. Then, we expand $H\left(g ; \phi_{2}-\phi_{1}\right)$ and apply Lemma 5.1 to perform the $g$-integration, thereby reducing the expectation to an integration over $g_{1}$ :

$$
\begin{aligned}
& \left\langle\chi_{\lambda}\left(\gamma\left(\phi_{1}\right)^{-1} \gamma\left(\phi_{2}\right)\right)\right\rangle \\
& \quad=\frac{1}{Z_{0}} \int H\left(g_{1} ; \phi_{1}\right) \chi_{\lambda}(g) H\left(g ; \phi_{2}-\phi_{1}\right) H\left(g^{-1} g_{1}^{-1} ; 2 \pi-\phi_{2}\right) d g d g_{1} \\
& =\frac{1}{Z_{0}} \int H\left(g_{1} ; \phi_{1}\right) \chi_{\lambda}(g) \sum_{\mu} \chi_{\mu}(1) \chi_{\mu}(g) e^{-\mu(\mu+1)\left(\phi_{2}-\phi_{1}\right)} H\left(g^{-1} g_{1}^{-1} ; 2 \pi-\phi_{2}\right) d g d g_{1} \\
& =\frac{1}{Z_{0}} H\left(g_{1} ; \phi_{1}\right) \sum_{\mu} \chi_{\mu}(1) e^{-\mu(\mu+1)\left(\phi_{2}-\phi_{1}\right)} \sum_{v=|\lambda-\mu|}^{\lambda+\mu} \chi_{v}\left(g_{1}^{-1}\right) e^{-v(v+1)\left(2 \pi-\phi_{2}\right)} d g_{1} .
\end{aligned}
$$

Finally, changing variable from $g_{1}$ to $g_{1}^{-1}$ and applying Lemma 5.1 yields the corollary. As in the previous examples, a minor modification of this computation serves as the induction step in computing the expectation of arbitrarily many products of functions of such loops. In particular, the expectation of a function of the product of Wilson loops proves to be invariant under rotations about the north pole. 


\section{Conclusion}

In this paper, we have interpreted the Yang-Mills measure as measure $\mu$ on $\mathscr{A} / \mathscr{G}_{m}$. We have used the description of $\mathscr{A} / \mathscr{G}_{m}$ as a bundle over $\Omega G$ to calculate $\mu$. Restricted to the fibers, and relative to a special choice of origin in each fiber, $\mu$ is a Gaussian measure. By integrating over the fibers in $\mathscr{A} / \mathscr{G}_{m}$, we have shown that $\mu$ descends to the Wiener measure on $\Omega G$. As a result, every expectation for Yang-Mills on the two-sphere reduces to an expectation for a free theory of $\Omega G$ via an expectation for a purely quadratic theory of Lie-algebra-valued functions on the two-sphere. We have derived, for $G=S U(2)$, explicit formulae for the quantum expectations of a special class of gauge-invariant functions on $S^{2}$.

Acknowledgements. I would like to thank I. M. Singer for starting me on this inquiry, and, most of all, for his advice and inspiration along the way. E. Farhi, D. Freedman, D. Stroock, J. Mickelsson, D. Düsedau, M. Lauer and J. Oliveira all provided clues at various points, for which help I am grateful. I am indebted to the Department of Mathematics at MIT for support in aid of this research.

\section{References}

1. Atiyah, M. F., Jones, J. D. S.: Topological aspects of Yang-Mills theory. Commun. Math. Phys. 61, 97-118 (1978)

2. Singer, I. M.: Some remarks on the Gribov ambiguity. Commun. Math. Phys. 60, 7-12 (1978)

3. Moore, G., Nelson, P.: Measure for moduli. Nucl. Phys. B266, 58-74 (1986)

4. D'Hoker, E., Phong, D. H.: Multiloops amplitudes for the Bosonic Polyakov string. Nucl. Phys. B269, 205-234 (1986)

5. Gawedzki, K.: Yang-Mills theory as Schrödinger quantum mechanics on the space of gauge-group orbits. Phys. Rev. D26, 3593-3610 (1982)

6. Uhlenbeck, K. K.: Connections with $L^{p}$ bounds on curvature. Commun. Math. Phys. 83, 31-42 (1982)

7. Atiyah, M. F., Bott, R.: Yang-Mills on a Riemann surface. Phil. Trans. R. Soc. Lond. A308, 523-615 (1982)

8. Glimm, J., Jaffe, A.: Quantum physics. New York, Berlin, Heidelberg: Springer 1987

9. Fegan, H. D.: The heat equation on a compact Lie group. Trans. Am. Math. Soc. 246, 339-357 (1978)

Communicated by A. Jaffe 